\title{
ABORDAJE PSICOLÓGICO DEL COVID-19: UNA REVISIÓN NARRATIVA DE LA EXPERIENCIA LATINOAMERICANAd
}

Hernán Scholten $^{\mathrm{a} 1}{ }^{\mathbb{D}}$, Vanetza Quezada-Scholz ${ }^{\mathrm{b}}{ }^{\oplus}$, Gonzalo Salas ${ }^{\mathbb{C}}$,

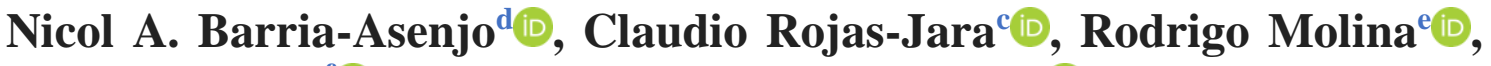
José E. García ${ }^{\mathrm{f}} \mathbb{D}$, María Teresa Julia Jorquera ${ }^{\mathrm{g}}{ }^{\mathbb{D}}$, Arturo Marinero Heredia $^{\mathrm{h}}{ }^{(}$, Alba Zambrano ${ }^{\mathrm{i}}$, Esteban Gómez Muzzio ${ }^{\mathrm{i}}$, Ariadna Cheroni Felitto ${ }^{\mathrm{k}}{ }^{\mathbb{D}}$, Tomás Caycho-Rodríguez ${ }^{1}$, Teresa ReyesGallardo $^{\mathrm{m}}{ }^{(\mathbb{B}}$, Nicolás Pinochet Mendoza $^{\mathrm{n}}{ }^{\mathbb{D}}$, Pitágoras José Binde ${ }^{\circ} \mathbb{C}^{-}$, Jamadier Esteban Uribe Muñoz ${ }^{\mathrm{P}}{ }^{\mathbb{D}}$, Johana Andrea Bernal Estupiñan ${ }^{q}$ \& $\mathrm{r}\left(\mathrm{D}, \boldsymbol{\&}\right.$ Francisco Somarriva ${ }^{\mathrm{e}}$.

Universidad de Buenos Aires, Buenos Aires, Argentina ; Universidad de Chile, Santiago, Chile ; Universidad Católica del Maule, Talca, Chile ; Cuadernos de Neuropsicología-Panamerican Journal of Neuropsychology, Santiago, Chiled, Colegio de Psicólogos de Chile, Santiago, Chile ${ }^{\mathrm{e}}$; Universidad Católica de Asunción, Asunción, Paraguay ; ; Universidad Central de Chile, La Serena, Chile ; Universidad Veracruzana, Xalapa, México $^{\mathrm{h}}$; Universidad de la Frontera, Temuco, Chile ${ }^{\mathrm{i}}$; Fundación América por la Infancia, Santiago, Chile ${ }^{\mathrm{j}}$; Universidad Católica del Uruguay, Montevideo, Uruguay ; Universidad Privada del Norte, Lima, Perúl; Universidad Santo Tomás, Osorno, Chile ${ }^{\mathrm{m}}$; Universidad Andrés Bello, Santiago, Chilen; Universidade Federal do Rio Grande do Norte, Natal, Brasil $^{\circ}$; Pontificia Universidad Católica de Valparaiso, Valparaiso, Chile ${ }^{\mathrm{p}}$; Universidad Santiago de Cali, Cali, Colombia ${ }^{\mathrm{q}}$; Fundación Universitaria Católica Lumen Gentium, Cali, Colombiar .

\section{RESUMEN}

Ante la rápida expansión del nuevo coronavirus (COVID-19) en todo el planeta, este artículo presenta un conjunto de propuestas elaboradas por psicólogas y psicólogos de América Latina que buscan abordar este fenómeno desde una perspectiva regional. En este sentido, se exponen tópicos como el problema de la sobreinformación y el impacto de los protocolos sanitarios sobre la salud mental en diversos ámbitos y sectores de la población. Se propone aquí, además de reunir algunos conocimientos aportados por investigaciones previas y difundir un conjunto de recursos para mitigar o paliar los efectos de la pandemia, contribuir con algunas reflexiones respecto del rol de psicólogas y psicólogos como profesionales de la salud en esta situación de crisis, así como apelar a los organismos nacionales e internacionales correspondientes.

Palabras Claves

COVID-19; psicología; Latinoamérica, salud mental

${ }^{1}$ Correspondence about this article should be addressed to Hernán Scholten: $\underline{\text { hsescholten@gmail.com }}$ 


\begin{abstract}
In view of the rapid expansion of the new coronavirus (COVID-19) throughout the planet, this article presents a set of proposals developed by Latin American psychologists who seek to address this phenomenon from a regional perspective. In this sense, topics such as the problem of overinformation and the impact of health protocols on mental health in different areas and sectors of the population are presented. It is proposed here, in addition to gathering some knowledge provided by previous research and disseminating a set of resources to mitigate or palliate the effects of the pandemic, to contribute with some reflections regarding the role of psychologists as health professionals in this critical situation, as well as to warn or appeal to the corresponding national and international organizations.
\end{abstract}

\title{
Keywords
}

COVID-19; psychology; Latin America; mental health 
PSYCHOLOGICAL APPROACH TO COVID-19: A NARRATIVE REVIEW OF THE LATIN AMERICAN EXPERIENCE

Una década después de la Gripe A (H1N1) estamos enfrentando la segunda pandemia global del siglo XXI: en diciembre de 2019 el nuevo coronavirus (COVID-19) comenzó su rápida expansión cobrando la vida de decenas de miles de personas en todo el planeta ${ }^{2}$. Las medidas dispuestas en la mayor parte de las naciones afectadas han puesto "en suspenso" nuestra vida cotidiana, nuestras agendas, nuestros hábitos, por un plazo que resulta difícil de determinar con certeza. Es posible afirmar, recurriendo a las categorías propuestas por Reinhardt Kosselleck (1993), que se ha abierto una amplia brecha entre nuestro espacio de experiencias y nuestro horizonte de expectativas. Los medios de comunicación masiva actualizan permanentemente las cifras de infectados y de víctimas fatales, e informan sobre las medidas que los estados nacionales disponen para enfrentar esta situación que diversos gobernantes han comparado con una guerra contra un enemigo o asesino invisible.

Estos protocolos dispuestos en numerosos países han impulsado algunos análisis inspirados en las indagaciones de Michel Foucault sobre las sociedades disciplinarias (Foucault, 1975, 2004; Scholten, 2013) y de Gilles Deleuze sobre las sociedades de control (Deleuze, 1995), los cuales ofrecen poderosas herramientas hermenéuticas de la coyuntura actual. Filósofos de la talla de Slavoj Žižek (2020) y Byung-Chul Han (2020) interpretan la escena contemporánea desde posturas antagónicas, proyectando escenarios contrapuestos a la luz del efecto de la pandemia y los protocolos sanitarios sobre los lazos comunitarios. En el espacio iberoamericano, un panorama similar nos muestra las propuestas de Atilio Borón (2020) y de Paul Preciado (2020) respecto del posicionamiento e incluso la supervivencia del sistema capitalista ante la coyuntura actual.

No obstante, muchas de estas elaboraciones teóricas no sólo presentan sus limitaciones para aplicarlas directamente a la situación latinoamericana, sino que tampoco proveen de herramientas concretas de intervención en un contexto saturado de incertidumbres, en el que se abren múltiples interrogantes sobre un tópico tan básico y fundamental como nuestras condiciones básicas de vida y nuestra propia supervivencia. En este sentido, resulta más útil rescatar las reflexiones sobre la psicología elaboradas por

\footnotetext{
${ }^{2}$ Según cifras del Coronavirus Resource Center de la John Hopkins University al día 17 de abril se registran 2.240.191 casos confirmados, 153.822 muertes y 568.343 recuperados. https://coronavirus.jhu.edu/map.html
} 
Nikolas Rose (1998, 1999) que, si bien comparten algunas de las limitaciones mencionadas anteriormente, nos permiten repensar desde una posición crítica un aspecto que usualmente es considerado como una falencia, un estigma o una "marca de nacimiento": la diversidad de la disciplina.

Aunque ha sido complejo establecer consensos sobre muchos aspectos, es esta diversidad la que ha posicionado a psicólogas y psicólogos como "expertos de la subjetividad" en el mundo moderno. El amplio abanico de saberes y prácticas de la psicología, como tecnología humana (Rose, 1998) abren la posibilidad de ofrecer diversos modos de operar en el espacio individual, familiar, grupal, institucional, educativo, laboral, jurídico, entre otros (Talak, Scholten, Macchioli, Del Cueto, \& Chayo, 2005).

De hecho, si bien en el combate contra la pandemia pueden resultar decisivas la intervención de la medicina y las políticas de los estados nacionales, el impacto de los protocolos implementados podría verse ampliamente beneficiado por los aportes de psicólogas y psicólogos, especialmente de quienes se desempeñan en los ámbitos más fuertemente afectados por la situación de crisis actual.

Con este fin, impulsados por el Colegio de Psicólogos de Chile, nos hemos reunido un conjunto de psicólogas y psicólogos latinoamericanos con el fin de formular una serie de propuestas que, a la luz de la crisis actual, buscan tomar en consideración las características regionales. En este sentido, para redactar este texto se han revisado las recomendaciones y los comunicados emitidos tanto por instituciones internacionales (Asociación Latinoamericana para la Formación y Enseñanza de la Psicología, 2020; Organización Mundial de la Salud [OMS], 2020a; OPS/OMS, 2020; Sociedad Interamericana de Psicología, 2020) como por centros académicos y profesionales locales (Coordinadora de Psicólogos del Uruguay, 2020; Facultad de Psicología de la Universidad de Buenos Aires, 2020; Facultad de Psicología de la Universidad de la República, 2020; Sociedad Chilena de Psicología Comunitaria, 2020). A su vez, se tuvieron en cuenta algunas publicaciones o proclamas colectivas realizadas por profesionales de nuestra disciplina, como el Manifiesto por la Psicología en Chile (Salas et al., 2019), redactado en el marco de una crisis social y política.

No buscamos solamente reunir algunos conocimientos obtenidos a partir de investigaciones internacionales, regionales o locales respecto de ciertos problemas específicos y difundir un conjunto de recursos con el fin de mitigar o paliar sus efectos. Intentamos también aportar algunas reflexiones que nos interpelan como ciudadanos y 
profesionales de la salud, y apelar a la intervención de los organismos públicos nacionales e internacionales correspondientes.

\section{Pandemia e información}

Lo que llamamos realidad, es el producto de complejos procesos psicológicos y psicosociales, vinculados con la necesidad de ordenar y otorgarle significado a nuestras experiencias cotidianas. En el mundo moderno, uno de los principales insumos que utilizamos para construir la realidad es la información provista por los diversos canales de noticias, que nos permiten recibir las novedades y comprender lo que sucede en nuestra ciudad, en nuestro país, en el continente y en el mundo.

En el transcurso del siglo XXI, se ha puesto en evidencia un fenómeno que los especialistas denominan sobrecarga informativa, infoxicación o infobesidad (Eppler \& Mengis, 2004), especialmente apreciable a partir de la veloz expansión de la internet, las redes sociales (e.g., Facebook, Twitter), los dispositivos móviles (e.g., smartphones, tablets) y las aplicaciones de mensajería (e.g, WhatsApp, Telegram), que se han integrado en la vida cotidiana de miles de millones de personas. Este fenómeno genera información en exceso, en un volumen que resulta imposible de asimilar y procesar, y bajo una modalidad de difusión que se preocupa más por capturar la atención de usuarios o por emocionarlos que por la veracidad de sus contenidos.

En la coyuntura actual, este fenómeno resulta claramente apreciable en tanto las noticias sobre el COVID-19 han monopolizado los medios masivos de comunicación. Las cifras de afectados y de muertos se actualizan permanentemente en las pantallas, se esbozan diversas teorías conspirativas y se anuncian porvenires apocalípticos, se proponen chivos expiatorios que estigmatizan a ciertos grupos étnicos o clases sociales, y circulan fake news (noticias falsas) de todo tipo. Los rumores se esparcen, cubriendo una necesidad cognitiva de explicar o dar sentido frente a la incertidumbre (Fernández Poncela, 2012). Algunos especialistas sostienen que es preciso enfocar este problema a partir de una falla en los filtros de información que utilizamos al publicar y compartir contenidos a través de redes sociales y aplicaciones de mensajería (Kear, 2011; Shirky, 2008).

Frente este panorama, es preciso señalar las consecuencias psicológicas y los efectos sobre la salud mental de este fenómeno que, si bien no es nuevo, se han vuelto particularmente evidentes en el marco de esta pandemia. Las investigaciones que se realizan desde hace más una década, además de efectos a mediano y largo plazo, muestran 
que la sobrecarga de información incrementa los niveles de estrés y ansiedad, y puede tener efectos nocivos en la autoestima (Bawden \& Robinson, 2009; Pantic, 2014; Sevinc \& D’Ambra, 2010).

Esto plantea la necesidad de modificar algunos hábitos sumamente arraigados en nuestras vidas cotidianas. En este sentido, una primera recomendación para reducir este impacto negativo de la sobrecarga de información es monitorear nuestro tiempo frente a la pantalla. Muchos dispositivos móviles tienen ya incorporada esta función y existen aplicaciones que pueden facilitar dicha tarea. Se sugiere que el uso de las redes sociales no debe exceder las dos horas diarias y que su utilización más de cinco horas por día tiene notorias consecuencias negativas.

En los últimos años, algunos especialistas e instituciones han comenzado a ofrecer recursos para llevar adelante una "desintoxicación digital" -traducción castellana de la expresión inglesa digital detox, que fue incorporado al diccionario de Oxford en 2012-, que incluye "retiros de silencio" y "terapias de desconexión" que duran entre siete días y seis meses (Levi, 2016; Syvertsen \& Enli, 2019).

\section{Pandemia y protocolos sanitarios}

La confirmación del primer caso de COVID-19 en América Latina, en febrero del 2020, llevó a que la región entrara en una fase de contención y se dispusieran protocolos sanitarios que incluyen medidas que van desde el cierre de fronteras, la cancelación de eventos masivos y el cierre de muchos comercios, hasta el aislamiento social o cuarentena, de especial interés aquí ya que fue implementada en muchos países de la región.

El establecimiento de esta medida, que preferimos denominar aislamiento sanitario $^{3}$, encuentra su justificación a partir de los riesgos que plantea la pandemia y su eficacia ya comenzó a recibir comprobación (Koo et al., 2020). En algunos casos, de hecho, resulta alarmante el desajuste entre las pautas y acciones propuestas por la Organización Mundial de la Salud y algunos gobiernos locales con respecto al aislamiento

\footnotetext{
${ }^{3}$ Por distintos canales comunicacionales se ha difundido que las personas deben mantener "distanciamiento social", lo cual es erróneo ya que lo que realmente se requiere, para evitar el contagio del virus, es un distanciamiento físico. Esta idea del distanciamiento social soslaya el aspecto psicológico que, paradójicamente, resulta fundamental para resistir el necesario confinamiento que implica la cuarentena: necesitamos consolidar e incluso reforzar el contacto social, compensando la distancia física a partir ya sea del recurso a las redes sociales virtuales o aplicaciones de mensajería y/o mediante la equilibrada interacción con el grupo con el que estemos compartiendo el confinamiento. Estas cuestiones serán retomadas y precisadas más adelante.
} 
sanitario, con argumentos que colocan a la economía por sobre la vida. Más allá de los posicionamientos ideológico-políticos, una obligación indiscutible del Estado es otorgarle prioridad y disponer todos sus recursos al cuidado de la salud y la vida de sus ciudadanas y ciudadanos.

Por ello, es preciso también tener en cuenta que las diversas medidas de aislamiento, basadas en el presupuesto de que todos somos potenciales infectados y propagadores de la enfermedad, tiene consecuencias a nivel psicológico que dependen de diversos factores. Entre ellos, además de la duración de la cuarentena, la frustración, el aburrimiento, los suministros e información inadecuada y las pérdidas financieras, se incluye el miedo a la infección (Brooks et al., 2020). En este sentido, diversos estudios sobre el cambio de comportamiento durante enfermedades infecciosas, muestran la importancia de evaluar la percepción del riesgo tanto en su dimensión cognitiva como afectiva para comprender el comportamiento de los individuos relacionado con las enfermedades infecciosas (Leppin \& Aro, 2009). Otros estudios indican que, durante las etapas tempranas de la enfermedad, las respuestas de riesgo afectivas pueden predecir mejor la presencia de comportamientos protectores (Karademas, Bati, Karkania, Georgiou, \& Sofokleous, 2013; Liao, Cowling, Lam, Ng, \& Fielding, 2010; Liao, Cowling, Lam, Ng, \& Fielding, 2014). Finalmente, algunos estudios recientes, han reportado los efectos de la preocupación relacionada con la enfermedad (PRE), como respuesta emocional a un padecimiento que influye en la adopción de comportamientos que promueven la salud, la prevención y el diagnóstico de diversas enfermedades (Bernat, Anderson, Parrish-Sprowl, \& Sparks, 2015; Keeton, Mikocka-Walus, \& Andrews, 2015; Ro, Lee, Kang, \& Jung, 2017). Por lo tanto, resulta importante indagar los niveles de la PRE relacionados con el COVID-19 a nivel local y regional, en tanto permitiría informar ciertos aspectos que necesiten de una intervención efectiva.

Otro fenómeno que también muestra el impacto de los protocolos sanitarios sobre los comportamientos es la denominada "violación de la cuarentena", la cual fue ampliamente difundida por los medios masivos de comunicación, dando a conocer las cifras de arrestos en diversos países de la región o publicando los vídeos de procedimientos policiales y la reacción de ciudadanas y ciudadanos ante quienes infringen las limitaciones establecidas por esta medida. Nuevamente vemos en esto el recurso al impacto emocional del espectador y la escasez de análisis respecto del fenómeno. En este sentido, consideramos que una herramienta heurística de gran valor puede ser la teoría de la disonancia cognoscitiva, propuesta por Leon Festinger (1975), 
cuyo ámbito de aplicación principal es el estudio de las actitudes. Diversos estudios recientes, muchos de ellos en el ámbito de la psicología social y la psicología política, se han inspirado en esta teoría para explicar comportamientos aparentemente irracionales o contradictorios (Abú Quevedo, 2005; García, 2012; Jablonski, Rodrigues, \& Assmar, 2005; Weiser, 1974) y permiten sugerir algunos lineamientos generales para analizar el caso de la actual pandemia -aunque es preciso reconocer que fue implementado desde un enfoque esencialmente individual que, a nivel de la estrategia de aproximación o intervención, atenta contra su celeridad y efectividad-.

Sin embargo, más allá de estas amplias referencias al efecto del aislamiento sanitario, nos interesa proponer una serie de reflexiones y recomendaciones a partir de diversas situaciones particulares, que pasaremos a revisar a continuación.

\section{El aislamiento sanitario y los vínculos}

La cuarentena impacta de manera directa en nuestros vínculos: a la incertidumbre respecto de la enfermedad y las restricciones temporales a nuestra libertad de circulación, se suma la distancia respecto de nuestras familias y amistades, lo cual puede generar altos montos de ansiedad o inducir estados depresivos en muchas personas.

Respecto de quienes se encuentran obligados a atravesar esta situación en soledad, es posible señalar sus consecuencias a partir de estudios previos sobre la soledad y aislamiento social como factores de riesgo (Holt-Lunstad et al., 2015). En este sentido, es preciso que quienes se encuentren en esta situación mantengan el contacto con familiares y amigos ya sea por vía telefónica o a través de las aplicaciones de mensajería, que puedan comunicar sus sensaciones y sentimientos, y organizar una rutina de actividades diarias que les permitan no sólo ocupar su tiempo sino también sentirse productivos (Rojas-Jara, 2020).

En el caso de aquellos que atraviesan la cuarentena acompañados por su pareja, también se constata un impacto en los vínculos: están obligados a una coexistencia que altera la rutina y pautas de convivencia habituales. Si bien no pueden descartarse las posibles consecuencias positivas de la cuarentena, como oportunidad para crear nuevos modos de vinculación o fortalecer los previos, es necesario advertir que esta nueva modalidad de convivencia puede exponer malestares latentes o incrementar malestares pre-existentes en los vínculos familiares. En este sentido, un dato a tener en cuenta son las noticias respecto de un notable incremento de divorcios en China, tras dos meses de 
cuarentena. Si bien esta noticia corresponde a una realidad socio-cultural con características particulares y amerita una indagación más rigurosa, debe servir de alarma a la luz de la situación imperante en muchos países de nuestra región.

Por otra parte, en tanto la forma más común de violencia de los hombres contra las mujeres es la infligida por la pareja -que en muchos casos debe compartir el aislamiento junto a su agresor-, nos interesa dar cuenta de nuestra preocupación por una problemática que la pandemia parece haber relegado a un segundo plano y que merece una especial consideración: la violencia de género. El Ministerio de la Mujer y Equidad de Género (Chile) y el Ministerio de las Mujeres, Géneros y Diversidad (Argentina), entre otros organismos, ya registraron un alarmante aumento en las llamadas a los servicios de asistencia a las mujeres en situación de violencia intrafamiliar. En este sentido, es preciso que, dentro de esta situación extraordinaria, los correspondientes organismos públicos locales y/o nacionales implementen protocolos que permitan a las víctimas presentar sus denuncias y recibir la correspondiente contención psicológica.

Además, los datos de una reciente encuesta a nivel regional muestran que en los hogares donde las mujeres sufren violencia infligida por su pareja, existe una mayor probabilidad de que los niños y niñas padezcan violencia física (Bott et al., 2019). Esto nos permite someter a consideración este sector de la población que, si bien no está incluida dentro de los grupos de riesgo que consideraremos más adelante, merece una especial atención.

\section{El aislamiento sanitario y la infancia}

La infancia en el Occidente moderno, como lo han mostrado diversos estudios, tiene una historia (Ariès, 1987; deMause, 1982; Sosenki \& Jackson Albarrán, 2012). Si hasta el siglo XVII no constituían una población claramente diferenciada del mundo de los adultos, niñas y niños son considerados actualmente como individuos cuyo derecho a un pleno desarrollo (físico, mental y social) y a expresar libremente sus opiniones es necesario garantizar y proteger (UNICEF, 2015).

En este sentido, es preciso considerar que, desde el comienzo de la cuarentena, ha cambiado de manera drástica la vida cotidiana de millones de niñas y niños de América Latina. Para madres, padres o tutores constituye todo un reto lograr que sus hijos se adapten a sus rutinas escolares, actividades extracurriculares y tiempo en casa ¿Qué hacer 
entonces ante la irrupción de un suceso de semejante magnitud, que nos anuncia que para estar a salvo debemos estar en aislamiento sanitario?

En este sentido, al reflexionar sobre el impacto de la pandemia de COVID-19 sobre la niñez, es preciso tener en cuenta dos pilares fundamentales de la salud mental infantil: la organización de la experiencia cotidiana y el logro de seguridad emocional (Cassidy \& Shaver, 2016; Solomon \& George, 2011; Zeanah, 2019). En sintonía con las recientes recomendaciones establecidas por el Colegio Oficial de la Psicología de Madrid (2020), es necesario señalar el rol crítico que madres, padres y otras figuras de apego cumplen en la protección y promoción de la resiliencia infantil en situaciones de fuerte adversidad como la que se está viviendo, mediante el despliegue de diversas competencias parentales (Gómez \& Contreras, 2019).

Las competencias vinculares posibilitan organizar el mundo emocional de niñas y niños, reduciendo el impacto del estrés tóxico en su salud mental (Shonkoff \& Phillips, 2000; Thomas, Letourneau, Campbell, Giesbrecht, \& Apron Study Team, 2018), apelando a la sensibilidad parental ante las necesidades emocionales de los niños (De Wolff \& Van Ijzendoorn, 1997), por ejemplo, acogiendo con paciencia las muestras de ansiedad y preocupación frente al encierro forzado, ofreciendo demostraciones claras de afecto (Zhou et al., 2002). Las competencias formativas apuntan a reducir la complejidad del mundo y organizan el aprendizaje cotidiano (Hoff, 2006), filtrando el exceso de información al que nos referimos previamente, regulando el tiempo de exposición a pantallas y explicando qué es el COVID-19 en términos adecuados a la edad del niño o niña, o incluso recurriendo al dibujo o al juego como estrategia. Las competencias protectoras organizan el ambiente y la rutina cotidiana para reducir las fuentes de estrés tóxico (Appleyard, Egeland, Dulmen, \& Sroufe, 2005), por ejemplo, haciendo que el día a día resulte en gran parte predecible, planificando una rutina familiar que establezca un balance, según las posibilidades de cada grupo familiar, entre las obligaciones (trabajo o estudio, alimentación, descanso) y el juego, que es un poderoso motor de la resiliencia familiar (Gómez \& Kotliarenco, 2010). También resulta útil generar medidas muy concretas de cuidado de la salud del grupo familiar como las que se han solicitado reiteradamente desde organismos internacionales de salud. Finalmente, las competencias reflexivas permiten organizarse a sí mismos como figuras de apego frente a las necesidades cotidianas de niñas y niños, y considerar al autocuidado como requisito para poder cuidar a otros (Rodrigo López, 2015; Swain, Lorberbaum, Kose, \& Strathearn, 2007). Se vuelve posible entonces que los adultos identifiquen aquellos aspectos 
subjetivos que pueden estar impactando negativamente sobre su rol de crianza y, de este modo, aumentar la probabilidad de desplegar una resiliencia parental y pedir ayuda cuando sea necesario (Gavidia-Payne, Denny, Davis, Francis, \& Jackson, 2015; Rodrigo López, 2015). La forma cómo padres, madres y/o cuidadores/as significativos manejen y ayuden a procesar la adversidad que sus hijas e hijos están experimentando durante estos meses, tendrá un impacto no sólo en la salud mental presente, sino que dejará huellas que se podrán rastrear incluso a la siguiente generación (Gray et al., 2017; Lieberman, Padron, Van Horn, \& Harris, 2005).

\section{Aislamiento sanitario y ámbito laboral}

La pandemia impactó de manera notoria en el espacio laboral, lo que nos lleva a considerar aquí algunas situaciones muy diversas. En lo que se refiere a las empresas -en todos los casos en que resulta posible- es preciso reemplazar la rutina laboral por modalidades de trabajo a distancia o teletrabajo, como el home office, que eviten la cercanía o contacto directo entre personas. Es necesario también consignar que estas modificaciones en el ámbito laboral, en muchos casos, se realiza de manera intempestiva y sin ninguna capacitación previa, lo cual tiene un impacto directo sobre trabajadoras y trabajadores.

En efecto, este cambio supone un escenario nuevo que en sí mismo puede resultar sumamente estresante y ansiógeno, a lo cual puede añadirse la dificultad de articular la rutina laboral con la dinámica y exigencias propias del entorno hogareño. En este sentido, dada la sensación de impredictibilidad e incontrolabilidad que trae consigo la propagación de la pandemia y el confinamiento (Quezada-Scholz, 2020), condiciones que tienen efectos cognitivos y psicológicos negativos como estrés y ansiedad (Mineka \& Kihlstrom, 1978), cobra relevancia crear contextos que otorguen un sentido de seguridad y estabilidad. Por tal razón, algunos especialistas recomiendan mantener la mayor parte de nuestros hábitos laborales, por ejemplo, ducharnos y vestirnos como lo hacíamos cotidianamente. También disponer de un espacio específico para trabajar al interior del hogar, evitando hacerlo en sillones o desde la cama. En otras palabras, es importante establecer una rutina con horarios y planificación de tareas diarias. Estos son recursos sumamente útiles para disminuir el estrés y la ansiedad entre las trabajadoras y los trabajadores, y les permitirá también sentirse productivos, ítem no despreciable en lo que se refiere a la salud mental. 
A su vez, es necesario tener en cuenta en este punto el derecho a la desconexión, que ya se incorporó al corpus legal en diferentes naciones (e.g., en Francia a partir del artículo 55 de la ley 1088/2016 y en España a partir de la Ley Orgánica 3/2018) y que comenzó a debatirse recientemente a nivel regional (Xiviller, 2020; Ministerio de Producción y Trabajo, 2019; Cámara de Diputados, 2017-2019). De este modo, se busca garantizar, fuera del tiempo de trabajo, el respeto de su tiempo de descanso, permisos y vacaciones, así como de su intimidad personal y familiar.

Es preciso considerar también a todos quienes están en la primera línea del combate contra la pandemia: los trabajadores de la salud. Se repite actualmente un escenario que ya ha sido documentado frente a los brotes de SARS y Ébola, la estigmatización y el rechazo (Bai et al., 2004; Brooks et al., 2020; Desclaux, Badji, Ndione, \& Sow, 2017). Diversos medios regionales registran la discriminación hacia profesionales de la medicina y la enfermería por parte de sus vecinos, temerosos del contagio, al mismo tiempo que, paradójicamente, se estableció como ritual el aplauso diario de agradecimiento a estos mismos profesionales.

Por otra parte, extensas horas de trabajo, un ambiente laboral inseguro, falta de los suministros necesarios para poder desempeñar sus funciones, e información confusa o ausente a la hora de afrontar esta emergencia sanitaria son factores estresantes que pueden llevar a los equipos de salud al burnout. En relación con esto, hay que tener en cuenta que el burnout no es sólo un fenómeno individual sino también grupal (Foladori, 2010), por lo que su prevención y tratamiento en los equipos de salud tienen que orientarse al trabajo con los equipos sanitarios en su totalidad, dando el espacio para elaborar el problema que están enfrentando y generar las instancias para poder lograr un funcionamiento grupal acorde a la tarea encomendada.

Finalmente, es necesario considerar también un panorama mucho más dramático, que son los casos en que la pandemia ha obligado al cierre de comercios o a la interrupción de las labores habituales, con la consecuente pérdida de ingresos que permitan sostener los insumos vitales básicos. En este caso, al temor y la incertidumbre generada por la pandemia se une la inseguridad económica, incrementado el impacto no solamente psicológico, sino también social y político de esta situación que afecta a una inmensa cantidad de la población regional ${ }^{4}$. En este sentido, nuestra recomendación se dirige

\footnotetext{
${ }^{4}$ Según la Organización Internacional del Trabajo (2018), en América Latina y el Caribe hay 140 millones de personas con trabajos informales.
} 
especialmente a los gobiernos nacionales, pero también a entidades privadas y organizaciones internacionales, para que presten la debida atención a esta situación e inicien acciones que permitan al menos aminorar su impacto presente y futuro.

\section{Aislamiento sanitario y poblaciones de riesgo}

Está comprobado que el COVID-19, por sus particulares características, resulta especialmente peligroso para ciertos sectores específicos de la población, para los cuales las condiciones del aislamiento o cuarentena requieren una contemplación particular (Center for Disease Control and Prevention, 2020).

Los adultos mayores constituyen un sector altamente vulnerable de nuestra población, que debe recibir especial consideración y atención. De hecho, en términos generales, el abordaje psicológico de este sector social también suele requerir un ajuste disímil a lo que puede pasar en personas jóvenes y adultas. A diferencia del resto de la población, los adultos mayores suelen ser especialmente susceptibles al aislamiento social y a los cambios en su rutina diaria, sobre todo aquellos que llegan a la vejez con una personalidad rígida (Salvarezza, 1988). La pérdida de la rutina o la dificultad para adoptar nuevos hábitos también tiene efectos en la estabilidad necesaria para que un adulto mayor se sienta vital y esperanzado. En este sentido, algunos estudios demuestran que los adultos mayores que mantienen sintomatología depresiva o ansiosa, ya sea solapada o explícita, puede sufrir un empeoramiento agudo por esta pandemia al subir su nivel de estrés y/o si es que se encuentran sin compañía de vecinos, amistades o familiares (Moutier, 2020).

En un plano complementario, es preciso considerar aquí el desafío que implica, en muchos casos, la inclusión digital de este sector de la población, con los problemas e incluso resistencias que a veces se presentan respecto del dominio de tecnologías que se incorporaron muy rápidamente a nuestra vida cotidiana y que revisten una particular importancia en la situación actual. Además de la situación económica, las dificultades cognitivas, sensoriales y motrices pueden obstaculizar la aproximación a un entorno que opera con lógicas nuevas y en gran medida ajena a los patrones de la era analógica. Entre otras consecuencias, esto podría llevar a una limitación o a la imposibilidad de acceder a los servicios de salud mental online, que son una modalidad predominante de asistencia en la coyuntura actual ( $\mathrm{Li}$ et al., 2020).

Por otra parte, un reciente documento publicado por la OMS considera también como población de riesgo a las personas en condición de encierro (OMS, 2020b). Esto 
permite apreciar el caso de quienes, por diversos motivos, se encuentran privadas de su libertad: personas adultas y jóvenes cumpliendo penas privativas de libertad o en centros de rehabilitación; niños, niñas y adolescentes en instituciones de protección; ancianos en geriátricos o personas con padecimientos mentales en hospitales o clínicas psiquiátricas ${ }^{5}$. Se trata de lugares frecuentemente olvidados, en donde diversas formas de violencia se manifiestan en sus formas más excesivas, y que sólo se vuelven centro de atención pública ante tensiones como las actualmente desatadas en diversas prisiones de la región frente al incremento de las medidas restrictivas adoptadas ante el COVID-19. Muchas de esas instituciones totales (Goffman, 1970) presentan condiciones estructurales deficitarias en lo que refiere a instalaciones, sobrepoblación, derecho a la salud, alimentación, educación, integridad física y mental y trato digno, para mencionar simplemente algunos aspectos.

En este marco, resulta fundamental adoptar una posición profesional que permita "rescatar" del olvido a aquellos que detrás de muros o a la intemperie son muchas veces consideradas como "descartables".

A efectos de no quedar entrampados entre construcciones sanitaristas y arbitrariedades punitivas, hoy más que nunca resulta indispensable integrar el conocimiento del Derecho Internacional de los Derechos Humanos a nuestra caja de herramientas conceptual y metodológica, así como permear el enfoque de derechos humanos de un saber "psi" que permite reconocer a cada sujeto en su singularidad irrepetible.

Ante el riesgo particularmente grave que significa el COVID-19 para aquellas personas constreñidas a vivir en estrecha proximidad, este posicionamiento profesional implica sumar nuestros saberes disciplinarios y nuestra voz a las instituciones nacionales e internacionales de defensa de los DDHH, para que en la grave crisis actual se efectivice la excepcionalidad y brevedad de la privación de libertad establecida en los tratados internacionales.

\footnotetext{
${ }^{5}$ A modo de ejemplo, en América Latina y el Caribe actualmente un millón y medio de personas se encuentran encarceladas. Según datos del World Prison Brief del 2018, la tasa de encarcelamiento en América Latina y el Caribe aumentó alrededor de un 28\%, principalmente entre las mujeres (52\%) y los jóvenes (Nuñovero Cisneros, 2019).
} 


\section{Cuarentena y consumo problemático de drogas}

Diversas investigaciones dan cuenta de la relación entre situaciones contextuales, como la cuarentena y el aislamiento, con diversas condiciones de salud mental como el estrés, la ansiedad y el consumo problemático de drogas (Alexander, 2012; Alexander, Coambs, \& Hadaway, 1978; Bloem, Bulten, \& Verkes, 2019; Gage \& Sumnall, 2018; Wang et al., 2011). Así, un factor contextual emergente y ansiógeno puede inducir a ciertas personas a presentar un consumo de drogas o este puede surgir como una modalidad de evasión frente a la angustia ante el encierro y la incertidumbre (Doyle, Shakeshaft, Guthrie, Snijder, \& Butler, 2019; Hall \& Weier, 2017; Rojas-Jara, 2020).

En esa línea, por ejemplo, una encuesta realizada a 549 empleados hospitalarios seleccionados al azar en Beijing (China) analizó el impacto psicológico tres años después del brote de SARS en el 2003 en dicha localidad, encontrándose síntomas de abuso o dependencia de alcohol que estaban asociados positivamente con la cuarentena o el trabajo en lugares de alto riesgo como las salas de SARS durante el brote (Wu et al., 2008). De este modo, es posible reconocer que las situaciones de estrés contextual por aislamiento, encierro o cuarentena generan una serie de consecuencias psicológicas que pueden ser abordadas a través del consumo de drogas, alcanzando estas una funcionalidad adaptativa -frente a una situación contextual displacentera- con mayor o menor éxito (Brooks et al., 2020; Eher, Rettenberger, \& Turner, 2019; Hadaway, Alexander, Coambs, \& Beyerstein, 1979; Rojas-Jara, 2018).

En consideración a lo previo, es identificable la conexión estrecha existente entre el aislamiento forzado por cuarentena, prisionización u otra condición contextual extraordinaria y el consumo de drogas (Brooks et al., 2020; Gomar, 2020; Lippi, Henry, Bovo, \& Sanchis). Esto implica el desafío de generar condiciones psicosociales positivas y alternativas que permitan a las personas enfrentar contextos forzosos de aislamiento y estrés a través de fuentes de adaptación diferentes (rutinas, hábitos, ejercicios, contacto, comunicación, etc.) que resten fuerza al uso de sustancias como exclusivo método de mitigación y faciliten el regreso a patrones conductuales normativos (Alexander, 2012; Johal, 2009; Rojas-Jara, 2019).

En el caso de consumidores problemáticos de drogas, el aislamiento o cuarentena puede llevar a una abstinencia forzada e incremento del deseo al no tener acceso al uso o provisión de la sustancia. Las personas en recuperación pueden ver ampliamente dificultado el acceso a los recursos terapéuticos (e.g., reuniones, grupos de rehabilitación 
o de autoayuda, etc.) y, por tanto, un aumento en la probabilidad de presentar una recaída en el consumo. En estos casos, es preciso garantizar algún tipo de seguimiento mediante consultas telefónicas, a través de aplicaciones de mensajería o video comunicaciones que les permitan acceder al apoyo psicológico que precisan.

\section{Después de la pandemia}

Las consecuencias de la actual pandemia no se limitan al presente como tampoco al corto plazo. Los aportes y recomendaciones de la psicología se extienden también a la situación que se presentará una vez que se atenúe esta condición y finalice el aislamiento sanitario vigente a nivel global. Es altamente probable que, a nivel individual, nos enfrentemos a situaciones que ya resultan conocidas como consecuencia ante situaciones de emergencias y desastres, tanto a nivel orgánico como a nivel cognitivo, emocional y comportamental. Estudios realizados a partir de sucesos como catástrofes naturales o atentados, coinciden en señalar que estos eventos aumentan la probabilidad de desarrollar trastornos de estrés post-traumático (Fraguas et al., 2006; Leiva-Bianchi, Baher \& Poblete, 2012). A su vez, la literatura disponible sobre el apoyo a víctimas de ese tipo de sucesos podría servir de guía para elaborar intervenciones que, desde la psicología, podrían realizarse en el marco del mundo post-coronavirus (García-Vera, Labrador, \& Larroy, 2008; Polanco-Carrasco, 2020).

Por otra parte, es necesario al menos anticipar el impacto de la pandemia cuando, al levantarse las medidas de aislamiento sanitario, se intente restaurar las rutinas habituales previas. Resulta imposible prever con exactitud este escenario, pero es preciso considerar las eventuales tensiones, e incluso resistencias que pueden presentarse. Quizá puedan surgir dificultades para restablecer la rutina anterior a la pandemia en el ámbito laboral y educacional, por una eventual habituación al ritmo del home office y de la educación a distancia, y será preciso evaluar el potencial impacto de este incremento de las relaciones remotas en la interacción social. Tal vez asistamos a la emergencia o construcción de una nueva normalidad, de nuevos hábitos y rutinas que requieran la intervención de la psicología y de los profesionales de la salud mental en general.

\section{A modo de conclusión}

La pandemia del COVID-19 es un evento muy reciente y la evidencia sobre sobre sus aspectos psicológicos, el impacto de las intervenciones y sus consecuencias está en pleno proceso de elaboración (Polanco-Carrasco, 2020). Sin embargo, como resultado del 
conjunto de reflexiones, propuestas y recomendaciones diversas que hemos formulado hasta este punto, entendemos que es también importante que aquellos que nos desempeñamos en el campo de la psicología extraigamos algunas enseñanzas de esta situación a partir de la experiencia previa del ejercicio de la psicología como profesión y de otros desastres naturales previos que han aquejado a nuestros países.

Es necesario reconocer que las indicaciones, consejos y herramientas que podemos proporcionar, aunque útiles, podrían perfeccionarse con una más amplia y exhaustiva investigación, especialmente a nivel local y regional. En este sentido, los saberes y prácticas psicológicas relacionadas con las situaciones de emergencias y desastres -como la psicología ambiental y la misma psicología de la emergencia- deben recibir una visibilidad y un apoyo con el que hasta ahora no han contado por parte de la propia comunidad de profesionales como de los poderes públicos y entidades privadas.

Del mismo modo, es imperativo que las organizaciones nacionales, regionales e internacionales establezcan y actualicen periódicamente los protocolos de formación profesional e intervención que permitan a psicólogas y psicólogos acceder a las herramientas para desarrollar, con la mayor eficacia posible, nuestro rol como agentes de la salud. En este sentido, un mayor énfasis en la alfabetización científica de los estudiantes de las carreras de salud -así como de los profesionales en general que toman decisiones en este campo- parece ser una tarea urgente a considerar para las próximas generaciones en formación (Polanco-Carrasco, 2016).

Estas variables y consideraciones previas pueden favorecer una reevaluación de nuestra disciplina por parte de las autoridades locales, nacionales e internacionales, como una voz científica y, por tanto, válida que merece ser escuchada a la hora de generar y establecer protocolos sanitarios y medidas de prevención de diversas patologías que afectan a nuestra población, como resulta ser el actual escenario global del COVID-19. 


\section{Referencias}

Abú Quevedo, J. (2005). La cuestión palestina: identidad nacional y acción colectiva. Madrid: Universidad Complutense de Madrid.

Alexander, B. K. (2012). Addiction: the urgent need for a paradigm shift. Substance Use \& Misuse, 47, 1475-1482. https://doi.org/10.3109/10826084.2012.705681

Alexander, B. K., Coambs, R., \& Hadaway, P. (1978). The effect of housing and gender on morphine self-administration in rats. Psychopharmacology, 58, 175-179. https://doi.org/10.1007/bf00426903

Appleyard, K., Egeland, B., Dulmen, M. H. M., \& Sroufe, L. A. (2005). When more is not better: the role of cumulative risk in child behavior outcomes. Journal of Child Psychology and Psychiatry, 46, 235-245. https://doi.org/10.1111/j.14697610.2004.00351.x

Ariès, Ph. (1987). El niño y la vida familiar en el antiguo régimen. Madrid: Taurus.

Asociación Latinoamericana para la Formación y Enseñanza de la Psicología (2020). Declaración. http://www.alfepsi.org/declaracion-alfepsi-covid19/

Bai, Y., Lin, C.C., Lin, C.Y., Chen, J.Y., Chue, C.M., \& Chou, P. (2004). Survey of stress reactions among health care workers involved with the SARS outbreak. Psychiatric Services, 55(9), 1055-1057. https://doi:10.1176/appi.ps.55.9.1055

Bawden, D., \& Robinson, L. (2009). The dark side of information: overload, anxiety and other paradoxes and pathologies. Journal of Information Science, 35(2), 180-191. https://10.1177/0165551508095781

Bernat, J. K., Anderson, L. B., Parrish-Sprowl, J., \& Sparks, G. G. (2015). Exploring the association between dispositional cancer worry, perceived risk, and physical activity among college women. Journal of American College Health, 63(3), 216220. https://doi.org/10.1080/07448481.2014.983927

Bloem, O., Bulten, E., \& Verkes, R. (2019). Changes in subjective wellbeing of prisoners on remand. International Journal of Prisoner Health, 15(2), 181-191. https://doi.org/10.1108/IJPH-01-2018-0003

Borón, A. (29 de marzo de 2020). La pandemia y el fin de la era neoliberal [Mensaje en un blog]. Atilio Borón. http://atilioboron.com.ar/la-pandemia-y-el-fin-de-la-eraneoliberal/

Bott, S., Guedes, A., Ruiz-Celis A., \& Mendoza, J. (2019). Intimate partner violence in the Americas: a systematic review and reanalysis of national prevalence estimates. Revista Panamericana de Salud Pública, 20(43), e26. https://doi.org/10.26633/RPSP.2019.26

Brooks, S. K., Webster, R. K., Smith, L. E., Woodland, L., Wessely, S., Greenberg, N., \& Rubin, G. J. (2020). The psychological impact of quarantine and how to reduce it: rapid review of the evidence. The Lancet, 395, 912-920. https://doi.org/10.1016/S0140-6736(20)30460-8

Cámara de Diputados (2017-2019). Modifica el Código del Trabajo y el Estatuto Administrativo para establecer el derecho a la desconexión digital de trabajadores de los sectores público y privado. https://www.camara.cl/legislacion/ProyectosDeLey/tramitacion.aspx?prmID=12 816\&prmBoletin=12284-13

Cassidy, J., \& Shaver, P. (2016). Hanbook of Attachment. Theory, research, and clinical aplications. Third Edition. London: The Guildford Press.

Center for Disease Control and Prevention (2020). People who are at higher risk for severe illnerss. https://www.cdc.gov/coronavirus/2019-ncov/need-extraprecautions/people-at-higher-risk.html 
Colegio Oficial de la Psicología de Madrid. (2020). Comunicado: Recomendaciones psicológicas para explicar a niños y niñas el brote de Coronavirus - Covid 19. https://www.copmadrid.org/web/comunicacion/noticias/1457/comunicadorecomendaciones-psicologicas-explicar-ninos-ninas-brote-coronaviruscovid-19

Coordinadora de Psicólogos del Uruguay (2020). Coyuntura: COVID-19. https://www.psicologos.org.uy/index.php/recomendaciones-covid19

De Wolff, M. S., \& Van Ijzendoorn, M. H. (1997). Sensitivity and attachment: A metaanalysis on parental antecedents of infant attachment. Child Development, 68(4), 571-591.

Deleuze, G. (1995). Conversaciones. 1972-1990. Valencia: Pre-textos.

deMause, Ll. (1982). Historia de la infancia. Barcelona: Alianza.

Desclaux, A., Badji, D., Ndione, A. G., \& Sow, K. (2017). Accepted monitoring or endured quarantine? Ebola contacts' perceptions in Senegal. Social Science \& Medicine, 178, 38-45. https://doi.org/10.1016/j.socscimed.2017.02.009

Doyle, M., Shakeshaft, A., Guthrie, J., Snijder, M., \& Butler, T. (2019). A systematic review of evaluations of prison-based alcohol and other drugs use behavioral treatment for men. Australian and New Zealand Journal of Public Health, 43(2), 120-130. https://doi.org/10.1111/1753-6405.12884

Eher, R., Rettenberger, M., \& Turner, D. (2019). The prevalence of mental disorders in incarcelated contact sexual offenders. Acta Psychiatrica Scandinava, 139(6), 572581. https://doi.org/10.1111/acps.13024

Eppler, M., \& Mengis, J. (2004). The concept of information overload: a review of literature from organization science, accounting, marketing, mis, and related disciplines. Information Society, 20(5), 325-344. https://doi.10.1080/01972240490507974

Facultad de Psicología de la Universidad de Buenos Aires. (2020). Recomendaciones psicológicas para afrontar la pandemia. http://www.psi.uba.ar/institucional/agenda/covid_19/recomendaciones_psicologi cas.pdf

Facultad de Psicología de la Universidad de la República (2020). Cuidado comunitario Subtítulo Frente al Coronavirus COVID-19. https://psico.edu.uy/covid/cuidado$\underline{\text { comunitario }}$

Fernández Poncela, A. M (2012). Psicología de masas, identidad social, epidemias y rumores la influenza en México. Sociológica, 27(76), 189-230.

Festinger, L. (1975). Teoría de la disonancia cognoscitiva. Madrid: Instituto de Estudios Políticos.

Foladori, H. (2010). Sufrimiento institucional: el burn-out en los equipos de salud. En Bilbao, A. y Morlans, I. (Eds), Duelo, pérdida y separación: figuras del sufrimiento humano (pp. 179-188). Valparaíso: Pontificia Universidad Católica de Valparaíso.

Fraguas, R., Gonsalves Henriques, S., De Lucia, M., Iosifescu, D., Schwartz, F., Rossi Menezes, P., Farid Gattaza, W., \& Arruda Martinse, M. (2006). The detection of depression in medical setting: a study with PRIME-MD. Journal of Affective Disorders, 91(1), 11-17. https://doi.org/10.1016/j.jad.2005.12.003

Foucault, M. (1975). Surveiller et punir. Naissance de la prison. Paris: Gallimard.

Foucault, M. (2004). Sécurité, territoire, population. Paris: Gallimard/Seuil.

Gage, S., \& Sumnall, H. R. (2018). Rat park: how rat paradise changes the narrative of addiction. Addiction, 114(5), 917-922. https://doi.org/10.1111/add.14481

García, J. E. (2012). El juicio político a Fernando Lugo y el poder explicativo de la disonancia cognoscitiva. Cuadernos Pedagógicos, 11-19. 
García-Vera, M.P., Labrador, F. J., \& Larroy, C. (2008). Ayuda psicológica a las víctimas de atentados y catástrofes. Guía de autoayuda y pautas de intervención psicológica elaboradas tras los atentados del 11-M. Madrid: Editorial Complutense.

Gavidia-Payne, S., Denny, B., Davis, K., Francis, A., \& Jackson, M. (2015). Parental resilience: a neglected construct in resilience research. Clinical Psychologist, 19(3), 111-121. https://doi.org/10.1111/cp.12053

Goffman, E. (1970). Internados. Ensayos sobre la situación social de los enfermos mentales. Buenos Aires: Amorrortu.

Gómez, E., \& Contreras, L. (2019). Manual escala de parentalidad positiva E2P v2. Fundación América por la Infancia: Santiago de Chile.

Gómez, E., \& Kotliarenco, M.A. (2010). Resiliencia familiar: un enfoque de investigación e intervención con familias multiproblemáticas. Revista de Psicología, 19(2), 103-131. https://doi.org/10.5354/0719-0581.2011.17112

Gray, S. A., Jones, C. W., Theall, K. P., Glackin, E., \& Drury, S. S. (2017). Thinking across generations: unique contributions of maternal early life and prenatal stress to infant physiology. Journal of the American Academy of Child \& Adolescent Psychiatry, 56(11), 922-929. https://doi.org/10.1016/j.jaac.2017.09.001

Hadaway, P., Alexander, B. K., Coambs, R., \& Beyerstein, B. (1979). The effect of housing and gender on preference for morphine-sucrose solutions in rats. Psychopharmacology, 66(1), 87-91. https://doi.org/10.1007/bf00431995

Hall, W., \& Weier, M. (2017). Lee Robins' studies of heroin use among US Vietnam veterans. Addiction, 112(1), 176-180. https://doi.org/10.1111/add.13584

Hoff, E. (2006). How social contexts support and shape language development. Developmental Review, 26, 55-88. https://doi.org/10.1016/j.dr.2005.11.002

Han, B-Ch. (22 de marzo de 2020). La emergencia viral y el mundo de mañana. El País, pp. s/p. https://elpais.com/ideas/2020-03-21/la-emergencia-viral-y-el-mundo-demanana-byung-chul-han-el-filosofo-surcoreano-que-piensa-desde-berlin.html

Holt-Lunstad, J., Smith, T. B., Baker, M., Harris, T., \& Stephenson, D. (2015). Loneliness and social isolation as risk factors for mortality: a meta-analytic review. Perspectives on Psychological Science, 10(2), 227-237. https://doi.org/10.1177/1745691614568352

Jablonski, B., Rodrigues, A., \& Assmar, E. M. L. (2005). Social-psychology and the invasion of Iraq. Revista de Psicología Social, 20(3), 387-398.

Johal, S. (2009). Psychosocial impacts of quarantine during disease outbreaks and interventions that may help to relieve strain. The New Zealand Medical Journal, 122(1296), 47-52.

Karademas, E. C., Bati, A., Karkania, V., Georgiou, V., \& Sofokleous, S. (2013). The association between pandemic influenza A (H1N1) public perceptions and reaction: a prospective study. Journal of Health Psychology, 18(3), 419-428. https://doi.org/10.1177/1359105312436765

Kear, K. (2011). Online and social networking communities: a best practice guide for educators. New York \& London: Routledge Press.

Keeton, R. L., Mikocka-Walus, A., \& Andrews, J. M. (2015). Concerns and worries in people living with inflammatory bowel disease (IBD): A mixed methods study. Journal of Psychosomatic Research, 78(6), 573-578. https://doi:10.1016/j.jpsychores.2014.12.004

Koo, J. R., Cook, A., Park, M., Sun, Y., Sun, H., Lin, J. T., Tam, C., \& Dickens, B. L. (2020). Interventions to mitigate early spread of SARS-CoV-2 in Singapore: a 
modelling study. The Lancet Infectious Diseases. https://doi.org/10.1016/S14733099(20)30162-6

Kosselleck, R. (1993). Futuro/Pasado. Barcelona: Paidós.

Leiva-Bianchi, M., Baher, G., \& Poblete, C. (2012). The effects of stress coping strategies in post-traumatic stress symptoms among earthquake survivors: an explanatory model of post-traumatic stress. Terapia Psicológica, 30(2), 51-59. https://dx.doi.org/10.4067/S0718-48082012000200005

Leppin, A., \& Aro, A. R. (2009). Risk perceptions related to SARS and avian influenza: theoretical foundations of current empirical research. International Journal of Behavioral Medicine, 16(1), 7-29. https://doi.org/10.1007/s12529-008-9002-8

Levi, D. M. (2016). Mindfulness digital: cómo aportar equilibrio a nuestras vidas digitales. Barcelona: Plataforma.

Li, W., Yang, Y., Liu, Z.H., Zhao, Y.J., Zhang, Q., Zhang, L., Cheung, T., Xiang, Y.T. (2020). Progression of Mental Health Services during the COVID-19 Outbreak in China. International Journal of Biological Sciences, 16(10), 1732-1738. https://doi.org/10.7150/ijbs.45120

Liao, Q., Cowling, B., Lam, W. T., Ng, M. W., \& Fielding, R. (2010). Situational awareness and health protective responses to pandemic influenza A (H1N1) in Hong Kong: a cross-sectional study. PLoS One, 5(10). e13350. https://doi.org/10.1371/journal.pone.001335

Liao, Q., Cowling, B. J., Lam, W. W., Ng, D. M., \& Fielding, R. (2014). Anxiety, worry and cognitive risk estimate in relation to protective behaviors during the 2009 influenza $\mathrm{A} / \mathrm{H} 1 \mathrm{~N} 1$ pandemic in Hong Kong: ten cross-sectional surveys. BMC Infectious Diseases, 14(1), 169. https://doi.org/10.1186/1471-2334-14-169

Lieberman, A., Padron, E., Van Horn. P. \& Harris, W. (2005). Angels in the nursery: The intergenerational transmission of benevolent influences. Infant Mental Health Journal, 26(6), 504-520. https://doi.org/10.1002/imhj.20071

Lippi, G., Henry, B., Bovo, C., \& Sanchis-Gomar, F. (2020). Health risk and potential remedies during prolonged lockdowns for coronavirus disease 2019 (COVID-19). Diagnosis. https://doi.org/10.1515/dx-2020-0041

Mineka, S., \& Kihlstrom, J. (1978). Unpredictable and uncontrollable events: a new perspective on experimental neurosis. Journal of Abnormal Psychology, 87, 256271. https://doi.org/10.1037//0021-843x.87.2.256

Ministerio de Producción y Trabajo (2019). Teletrabajo en Argentina: primer libro blanco nacional de buenas prácticas en teletrabajo, trabajo remoto y trabajo conectado. Buenos Aires: Secretaría de Gobierno de Trabajo y Empleo.

Moutier, Ch. (2020). COVID-19: We must care for older adults' mental health. https://afsp.org/covid-19-we-must-care-for-older-adults-mental-health/

Nuñovero Cisneros, L. (2019). Cárceles en América Latina 2000-2018. Tendencias y desafíos. Lima: Departamento Académico de Ciencias Sociales - Pontificia Universidad Católica de Perú. http://repositorio.pucp.edu.pe/index/handle/123456789/169206

Organización Mundial de la Salud (2020a) Preguntas y respuestas sobre la enfermedad por

coronavirus

(COVID-19).

https://www.who.int/es/emergencies/diseases/novel-coronavirus-2019/advicefor-public/q-a-coronaviruses

Organización Mundial de la Salud (2020b). Preparedness, prevention and control of COVID-19 in prisons and other places of detention. http://www.euro.who.int/data/assets/pdf_file/0019/434026/Preparednessprevention-and-control-of-COVID-19-in-prisons.pdf 
Organización Internacional del Trabajo (2018). OIT: Cerca de 140 millones de trabajadores en la informalidad en América Latina y el Caribe. https://www.ilo.org/americas/sala-de-prensa/WCMS_645596/lang--es/index.htm

OPS/OMS (2020). Consideraciones psicosociales y de salud mental durante el brote de COVID-19. $\quad$ https://www.paho.org/es/documentos/consideracionespsicosociales-salud-mental-durante-brote-covid-19

Pantic, I. (2014). Online social networking and mental health. Cyberpsychology, Behavior and Social Networking, 17(10), 652-657. https://doi.org/10.1089/cyber.2014.0070

Preciado, P. (28 de marzo de 2020). Aprendiendo del virus. El País, pp. s/p. https://elpais.com/elpais/2020/03/27/opinion/1585316952_026489.html

Polanco-Carrasco, R. (2020). Cuando la ciencia de la conducta salva vidas. Cuadernos de Neuropsicología/Panamerican Journal of Neuropsychology, 14(1), 8-9. https://doi.org/10.7714/CNPS/14.1.101

Polanco-Carrasco, R. (2016). La publicación científica como placebo. Cuadernos de Neuropsicología/Panamerican Journal of Neuropsychology, 10(2), 8-12. https://doi.org/10.7714/CNPS/10.2.101

Quezada-Scholz, V, E. (2020). Miedo y psicopatología: la amenaza que oculta el COVID19. Cuadernos de Neuropsicología/Panamerican Journal of Neuropsychology, 14(1), 19-23. https://doi.org/10.7714/CNPS/14.1.202

Ro, J. S., Lee, J. S., Kang, S. C., \& Jung, H. M. (2017). Worry experienced during the 2015 Middle East respiratory syndrome (MERS) pandemic in Korea. PloS One, 12(3), e0173234. https://doi.org/10.1371/journal.pone.0173234

Rodrigo López, M. J., (coord.) (2015). Manual práctico de parentalidad positiva. Madrid: Síntesis.

Rojas-Jara, C. (2018). Innovation in the field of drugs: the need to rethink the use, the user, and the psychological treatment. En J.C. Penagos-Corso, \& M.A. Padilla (Eds.), Challenges in creativity \& psychology for the XXI century (pp. 259-271). México: UDLAP/Universidad de Guadalajara.

Rojas-Jara, C. (2019). Drogas, drogos y drogodependencias: reformulando el objeto, el sujeto y el tratamiento psicológico del consumo problemático de drogas. En C. Rojas-Jara (Ed), Drogas: sujeto, sociedad y cultura (pp.107-122). Talca: Nueva Mirada Ediciones.

Rojas-Jara, C. (2020). Cuarentena, aislamiento forzado y uso de drogas. Cuadernos de Neuropsicología/Panamerican Journal of Neuropsychology, 14(1), 24-28. https://doi.org/10.7714/CNPS/14.1.203

Rose, N. (1998). Inventing our selves: psychology, power, and personhood. Cambridge: Cambridge University Press.

Rose, N. (1999). Governing the soul: the shaping of the private self. Londres: Free Association Books.

Salas, G., Urzúa, A., Larraín, A., Zúñiga, C., Cornejo, M., Sisto, V., Zambrano Constanzo, A., Urra, M., Polanco-Carrasco, R., Caqueo-Urizar, A., Pérez-Salas, C.P., Acuña Mercier, P., \& Kühne, W. (2019). Manifiesto por la Psicología en Chile: A propósito de la revuelta del 18 de octubre de 2019. Terapia Psicológica, 37(3), 317-326. https://doi.org/10.4067/S0718-48082019000300317

Salvarezza, L. (1988) Psicogeriatría. Teoría y clínica. Buenos Aires: Editorial Paidós.

Scholten, H. (2013). Disciplina, biopolítica y gobierno. En Ruvituso, M. (coord.), Cuadernos de pensamiento biopolítico latinoamericano (pp. 97-103). Buenos Aires: UNIPE Editorial Universitaria. 
Sevinc, G., \& D'Ambra, J. (7-9 de junio de 2010). The influence of self-esteem and locus of control on perceived email overload. 18th European Conference on Information Systems, ECIS 2010, Pretoria, Sudáfrica.

Shirky, C. (2008). It's not information overload. It's filter failure. https://www.mascontext.com/issues/7-information-fall-10/its-not-informationoverload-its-filter-failure/

Shonkoff, J., \& Phillips, D. (Eds) (2000). From neurons to neighborhoods: the science of early childhood development. Washington D.C.: National Academy Press.

Sociedad Interamericana de Psicología (2020). Llamado de la SIP ante la pandemia de coronavirus. https://sipsych.org/covid/?lang=es

Sociedad Chilena de Psicología Comunitaria (2020). La crisis social de la pandemia en Chile. https://www.agenciadenoticias.org/la-crisis-psicosocial-de-la-pandemiaen-chile/

Solomon, J., \& George, C. (2011). Disorganized attachment and caregiving. New York: The Gilford Press.

Sosenki, S., \& Jackson Albarrán, E. (2012). Nuevas miradas a la historia de la infancia en América Latina: entre prácticas y representaciones. México: UNAM Instituto de Investigaciones Históricas.

Swain, J. E., Lorberbaum, J. P., Kose, S., \& Strathearn, L. (2007). Brain basis of early parent-infant interactions: psychology, physiology, and in vivo functional neuroimaging studies. Journal of Child Psychology and Psychiatry, 48(3-4), 262287. https://doi.org/10.1111/j.1469-7610.2007.01731.x

Syvertsen, T., \& Enli, G. (2019). Digital detox: media resistance and the promise of authenticity. Convergence: The International Journal of Research into

New Media Technologies, 1-15. https://doi.org/10.1177/1354856519847325

Talak, A. M., Scholten, H., Macchioli, F., Del Cueto, J., \& Chayo, Y. (2005). Novedad y relevancia en la historia del conocimiento psicológico. Anuario de investigaciones, 12, 305-313.

Thomas, J. C., Letourneau, N., Campbell, T. S., Giesbrecht, G. F., \& Apron Study Team. (2018). Social buffering of the maternal and infant HPA axes: mediation and moderation in the intergenerational transmission of adverse childhood experiences. Development and Psychopathology, 30(3), 921-939. https://doi.org/10.1017/S0954579418000512

UNICEF (2015). Convención de los derechos del niño. https://www.unicef.es/sites/unicef.es/files/comunicacion/ConvencionsobrelosDe rechosdelNino.pdf

Wang, Y., Xu, B., Zhao, G., Cao, R., He, X., \& Fu, S. (2011). Is quarantine related to immediate negative psychological consequences during the 2009 H1N1 epidemic? General Hospital Psychiatry, 33(1), 75-77. https://doi.org/10.1016/j.genhosppsych.2010.11.001

Weiser, N. (1974). The effects of prophetic disconfirmation of the committed. Review of Religious Research, 16(1), 19-30.

Wu, P., Liu, X., Fang, Y., Fan, B., Fuller, C. J., Guan, Z., Yao, Z., Lu, J., Litvak, I. J. (2008). Alcohol abuse/dependence symptoms among hospital employees exposed to a SARS outbreak. Alcohol and Alcoholism, 43(6), 706-712. https://doi.org/10.1093/alcalc/agn073.

Xiviller, Ch. (13 de abril de 2020). El derecho a la desconexión digital en tiempos de cuarentena. El Observador. https://www.elobservador.com.uy/nota/el-derecho-ala-desconexion-digital-en-tiempos-de-cuarentena-2020413503. 
Zeanah, Ch. (2019). Handbook of Infant Mental Health (Fourth Edition). The Guilford Press: New York.

Zhou, Q., Eisenberg, N., Losoya, S., Fabers, R., Reiser, M., Guthrie, I., Murphy, B., Cumberland, A., \& Shepard, S. (2002). The relations of parental warmth and positive expressiveness to children's empathy-related responding and social functioning: a longitudinal study. Child Development, 73(3), 893-915.

Žižek, S. (2020). Pandemic. Covid-19 shakes the world. New York: O/R Books.

Received: 2020/04/20

Accepted: 2020/05/05 